\title{
Parametric analysis of the strain-dependent behavior of a metamaterial electric resonator
}

\author{
B. Arritt, ${ }^{1,2, a)}$ B. Adomanis, ${ }^{1}$ T. Khraishi, ${ }^{2}$ and D. Smith ${ }^{3}$ \\ ${ }^{1}$ Air Force Research Laboratory/Space Vehicles Directorate, Kirtland AFB, New Mexico 87117, USA \\ ${ }^{2}$ Department of Mechanical Engineering, University of New Mexico, Albuquerque, New Mexico 87131, USA \\ ${ }^{3}$ Department of Electrical and Computer Engineering, Duke University, Durham, North Carolina \\ 27708, USA
}

(Received 18 September 2010; accepted 11 October 2010; published online 10 November 2010)

\begin{abstract}
In this paper, we describe the strain-dependent behavior of an electric-LC (ELC) resonator unit cell, commonly used in metamaterial designs. We leverage analytic expression to understand the way strain manifests itself in a change in electromagnetic (EM) response. We verify the simplified physical models using full-wave simulations and generalize the trends to accommodate the strain profile for any arbitrary plane-stress loading scenario. (c) 2010 American Institute of Physics.
\end{abstract}

[doi:10.1063/1.3507892]

Metamaterials can greatly expand man's ability to control interactions with electromagnetic radiation and enable such phenomena as cloaking, ${ }^{1,2}$ beyond diffraction-limited imaging, ${ }^{3}$ gradient negative-index lenses, ${ }^{4}$ and perfect absorbers. ${ }^{5}$ They are a powerful concept by allowing designers to utilize geometry, and not just material properties, to engineer a structure's electromagnetic response; often providing properties not found in nature.

However, transitioning metamaterials into real, operational systems requires knowledge of their behavior in relevant environments. Of significance is the role mechanical loading/strain plays in the electromagnetic response of a metamaterial. Mechanical strain is by definition, a deformation of the geometry of a structure. Since metamaterials rely so heavily on geometry for the desired response, it implies a direct causal relationship between applied strain and electromagnetic performance.

Previous efforts investigated the strain ${ }^{6}$ and temperature ${ }^{7}$ dependent response of magnetic resonant elements; Melik et al. $^{6}$ even proposes using metamaterials as wireless strain gauges. Our efforts focused on a critical missing piece, the electric-LC resonator, depicted in Fig. 1. This structure operates at $\mathrm{x}$-band, utilizing two parallel capacitors for enhanced resonant response. Figure 1 depicts the S-parameter curves for the cell.

The mechanical model assumes the metamaterial unit cell is part of a large $(>10 \lambda)$, load-bearing structure. Mechanical loading on the cell is homogeneous, and the copper contributes insignificantly to the overall stiffness of the composite; therefore, the in-plane strain profile is approximately uniform across the unit cell (no local stiffening effects from the adhered copper). Due to the uniformity of the strain profile and the resultant absence of higher order differential terms, the linear system of Eq. (1) can be utilized to describe the deformed geometry of the unit cell as follows: ${ }^{8}$

$$
\left\{\begin{array}{c}
X^{1} \\
Y^{1} \\
Z^{1}
\end{array}\right\}=\left[\begin{array}{lll}
E_{X X} & E_{X Y} & E_{X Z} \\
E_{X Y} & E_{Y Y} & E_{Y Z} \\
E_{X Z} & E_{Y Z} & E_{Z Z}
\end{array}\right]\left\{\begin{array}{c}
X^{0} \\
Y^{0} \\
Z^{0}
\end{array}\right\}+\left\{\begin{array}{c}
X^{0} \\
Y^{0} \\
Z^{0}
\end{array}\right\} .
$$

\footnotetext{
${ }^{a)}$ Electronic mail: brandon.arritt@kirtland.af.mil.
}

The superscript 0 and 1 refer to the undeformed and deformed geometries, respectively, and the $3 \times 3$ matrix is the mechanical strain tensor. The model accommodates different values of $E_{Z Z}$ in the substrate and copper, due to the differing mechanical properties in the materials. Additionally, the analysis was restricted to plane stress because $\mathrm{e}^{1,2}$ demonstrated that changes in EM performance due to curvature in the unit cell can be neglected. Therefore, terms $E_{X Z}$ and $E_{Y Z}$ are equal to zero.

The unit cell was modeled in ANSYS-HFSS, ${ }^{9}$ utilizing equation surfaces that integrate the strain tensor transformation Eq. (1) into the surface descriptions. Thus the model accommodates geometry changes resulting from any arbitrary plane-stress loading scenario. Analysis was accomplished by running parametric sweeps of $E_{X X}, E_{X Y}, E_{Y Y}, E_{Z Z S}$, and $E_{Z Z C}$; where $S$ and $C$ in the subscript refer to the substrate and copper, respectively. Each strain component was evaluated between $-5 \%$ and $+5 \%$, with the influence of each strain component evaluated separately.

The model demonstrated that out-of-plane normal strains (affecting the thickness of the copper and substrate) had a negligible effect. However, the in-plane strains $\left(E_{X X}, E_{X Y}\right.$, and $\left.E_{Y Y}\right)$ do produce an effect, as depicted in the Fig. 2.

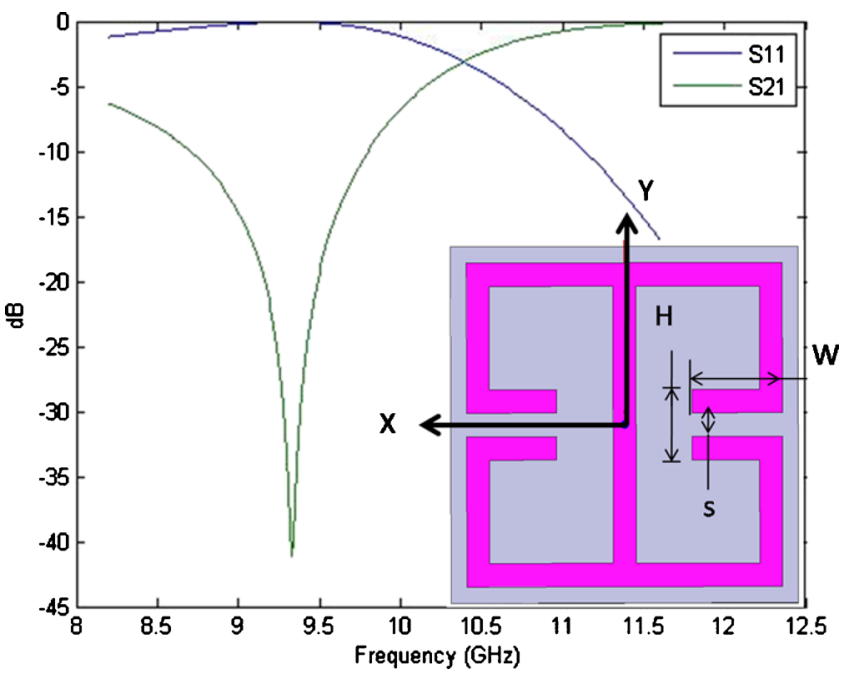

FIG. 1. (Color online) Unit cell and baseline/unloaded S-Parameter data. 


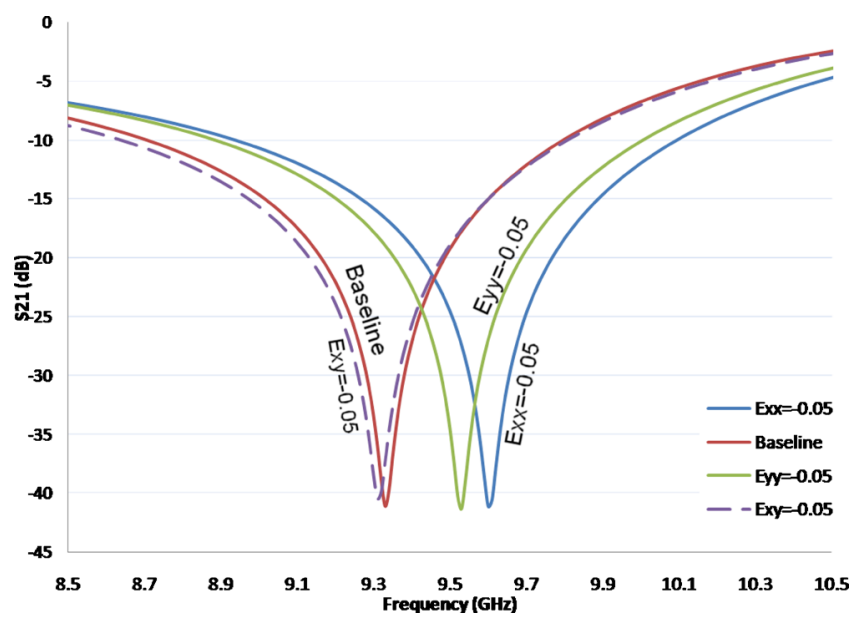

FIG. 2. (Color online) Transmission (S21) of unit cell at differing strain values.

Analytic expressions were leveraged to better understand the mechanisms for the strain-dependence. Equation (2) (Ref. 10) describe the capacitance (C) for a co-planar capacitor and Eq. (3) (Ref. 11) describes the self-inductance (L) of a thin, conducting strip. The total effective inductance and capacitance for the unit cell (accounting for addition of parallel and/or series capacitors and inductors, as well as interand intracell capacitances) were computed, and then applied to Eq. (4) (Ref. 12) to determine the cell's resonant frequency as a function of strain.

$$
\begin{aligned}
& C=C_{1}+C_{2}+C_{3}+C_{4}, \\
& C_{1}=\varepsilon_{0} \frac{2}{\pi} \ln \left(2 \alpha \frac{H}{s}\right) W, \\
& C_{2}=\varepsilon_{0} \frac{\varepsilon_{2}}{\frac{s}{h_{S}}+\frac{4}{\pi} \ln (\alpha)} W, \\
& C_{3}=\varepsilon_{0} \frac{h_{C}}{s} W, \\
& C_{4}=\varepsilon_{0} \frac{1}{\pi} \ln \left(2 \alpha \frac{H}{s}\right) W,
\end{aligned}
$$

$W, s$, and $H$ are depicted in Fig. 1. $\varepsilon_{2}$ is dielectric constant of the substrate, $h_{C}$ is thickness of the copper, $h_{S}$ is thickness of the substrate, and $\alpha=2$ (Ref. 13),

$$
L=2 l\left[\log 10\left(\frac{2 l}{b}\right)+\frac{1}{2}+\frac{b}{3 l}-\frac{b^{2}}{24 l^{2}}\right],
$$

$l$ is length and $b$ is width of the conducting strip.

$$
\omega_{0}^{\prime}=\frac{1}{\sqrt{L^{\prime} C^{\prime}}}=\frac{1}{\sqrt{L \frac{L^{\prime}}{L} C \frac{C^{\prime}}{C}}}=\omega_{0} \sqrt{\frac{L}{L^{\prime}} \frac{C}{C^{\prime}}},
$$

where the primes correspond to the strained unit cell and unprimed corresponds to the baseline/unstrained cell. Equations (2)-(4) produce a reasonable approximation of the resonant frequency of the baseline design (predicted value of $9.63 \mathrm{GHz}$, versus the modeled value of $9.33 \mathrm{GHz}$; error of

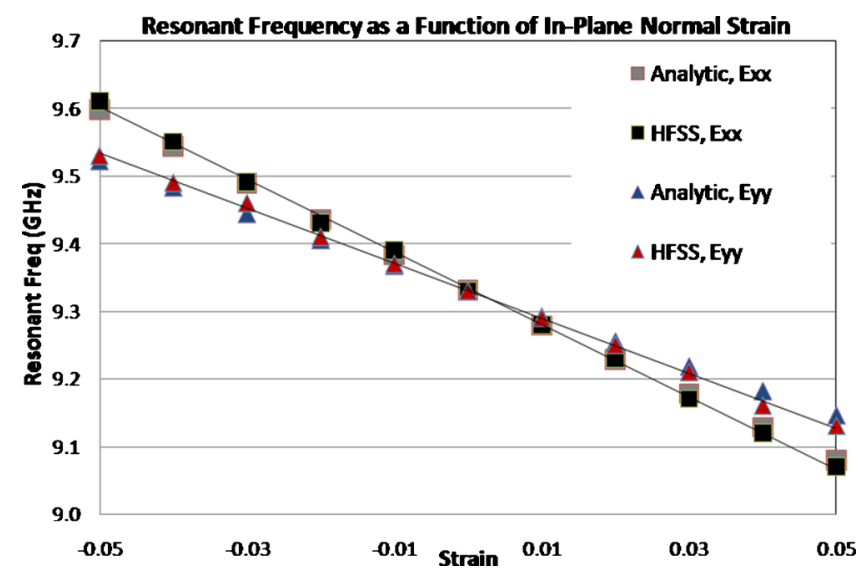

FIG. 3. (Color online) Resonant frequency as a function of the in-plane normal strains.

3.2\%). Additionally, these expressions allow accurate predictions of the shift in resonance, when compared to full-wave simulations in HFSS. Figure 3 depicts the resonant frequency as a function of applied normal strain, comparing the analytic predictions to the solutions of the full-wave simulation. As Fig. 3 shows, the analytic predictions and the simulation results for $E_{X X}$ and $E_{Y Y}$ are in strong agreement.

Referring back Fig. 1, one can qualitatively observe that a tensile strain (extension) in the $X$-direction causes an increase in both the cell's self-inductance [increases $l$ in Eq. (3)] and capacitance [increases $W$ in Eq. (2)], resulting in a decrease in the resonant frequency. A compressive strain causes the opposite affects. Conversely, a tensile strain in the $Y$-direction causes an increase in the cell's inductance [again by increasing $l$ in Eq. (3)] while decreasing the cell's capacitance [increasing $s$ in Eq. (2)]. A shear strain (irrespective of sign) causes a small increase in the cell's inductance (increases $l$ ) as well as a slight increase in the cell's capacitance (decreases $s$ ); resulting in a slight drop in the resonant frequency.

While the trends in Fig. 3 are not strictly linear [according to Eqs. (2)-(4)], they can be approximated by linear trends for the range of deformations pertinent to most mechanical systems. The linearity of the trends allows the resultant resonant frequency, for a metamaterial surface subjected to any complex plane-stress loading scenario (components of $E_{X X}, E_{X Y}$, and $E_{Y Y}$ ), to be accurately predicted via superposition of the influence from all of the constituent strain values. Thus, the strain-dependent resonant frequency for this particular unit cell is described by the following equation:

$$
f_{0}(G H z)=9.33-5.35 E_{X X}-4.06 E_{Y Y}-0.4\left|E_{X Y}\right|
$$

In summary, we report efforts to understand the straindependent behavior of metamaterial structures. Existing analytic expressions were leveraged to predict and understand the change in resonant frequency, and validated those predictions against full-wave simulations. The trends are linear over large ranges of strain, allowing the resonant frequency of a sample subjected to complex combined loads to be easily described through a linear superposition of the influence of each of the independent strain components. 
The authors wish to acknowledge financial and programmatic support from the U.S. Air Force Office of Scientific Research.

${ }^{1}$ D. Schurig, J. Mock, B. Justice, S. Cummer, J. Pendry, A. Starr, and D. Smith, Science 314, 977 (2006).

${ }^{2}$ S. Cummer, B.-I. Popa, D. Schurig, D. Smith, and J. Pendry, Phys. Rev. E 74, 036621 (2006).

${ }^{3}$ J. Pendry, Phys. Rev. Lett. 85, 3966 (2000).

${ }^{4}$ D. Smith, J. Mock, A. Starr, and D. Schurig, Phys. Rev. E 71, 036609 (2005).

${ }^{5}$ N. Landy, S. Sojuyigbe, J. Mock, D. Smith, and W. Padilla, Phys. Rev. Lett. 100, 207402 (2008).

${ }^{6}$ R. Melik, E. Unal, N. Perkgoz, C. Puttlitz, and H. Demir, Appl. Phys. Lett.
95, 011106 (2009).

${ }^{7}$ K. Boratay Alici and E. Ozbay, New J. Phys. 11, 043015 (2009).

${ }^{8}$ J. Lubliner, Plasticity Theory (Pearson Education, Upper Saddle River, NJ, 2006).

${ }^{9}$ Ansys, Inc., Southpointe, 275 Technology Dr., Canonsburg, PA 15317, USA.

${ }^{10}$ M. Vukadinovic, B. Malic, M. Kosec, and D. Krizaj, Meas. Sci. Technol. 20, 115106 (2009).

${ }^{11}$ E. B. Rosa, Bulletin of the Bureau of Standards (Washington Government Printing Office, Washington, D.C., 1908) Vol. 4, pp. 301-344.

${ }^{12}$ P. Lorrain, D. P. Corson, and F. Lorrain, Electromagnetic Fields and Waves, including Electric Circuits, 3rd ed. (W.H. Freeman, New York, 1988).

${ }^{13}$ O. G. Vendik, S. P. Zubko, and M. A. Nikol'skii, Tech. Phys. 44, 349 (1999). 